Research report

\title{
A lack of appetite for information and computation. Simple heuristics in food choice ${ }^{\text {is }}$
}

\author{
Michael Schulte-Mecklenbeck ${ }^{\mathrm{a}, \mathrm{b}, *}$, Matthias Sohn ${ }^{\mathrm{c}}$, Emanuel de Bellis ${ }^{\mathrm{d}}$, Nathalie Martin ${ }^{\mathrm{e}}$, Ralph Hertwig ${ }^{\mathrm{a}}$ \\ ${ }^{a}$ Center for Adaptive Rationality (ARC), Max Planck Institute for Human Development, Lentzeallee 94, 14195 Berlin, Germany \\ ${ }^{\mathrm{b}}$ Chair of Decision Theory and Behavioral Game Theory, ETH Zürich, Clausiusstrasse 50, 8092 Zürich, Switzerland \\ ${ }^{\mathrm{c}}$ Chair of Management Accounting, Bundeswehr University Munich, Werner-Heisenberg-Weg 39, 85577 Neubiberg, Germany \\ ${ }^{\mathrm{d}}$ Center for Customer Insight, University of St.Gallen, Bahnhofstrasse 8, 9000 St.Gallen, Switzerland \\ e Nestlé Research Center, Vers-Chez-Les-Blanc, Switzerland
}

\section{A R T I C L E I N F O}

\section{Article history:}

Received 9 January 2013

Received in revised form 7 August 2013

Accepted 12 August 2013

Available online 29 August 2013

\section{Keywords:}

Food choice

Heuristics

Process tracing

Rational choice

MouselabWeb

\begin{abstract}
A B S T R A C T
The predominant, but largely untested, assumption in research on food choice is that people obey the classic commandments of rational behavior: they carefully look up every piece of relevant information, weight each piece according to subjective importance, and then combine them into a judgment or choice. In real world situations, however, the available time, motivation, and computational resources may simply not suffice to keep these commandments. Indeed, there is a large body of research suggesting that human choice is often better accommodated by heuristics-simple rules that enable decision making on the basis of a few, but important, pieces of information. We investigated the prevalence of such heuristics in a computerized experiment that engaged participants in a series of choices between two lunch dishes. Employing MouselabWeb, a process-tracing technique, we found that simple heuristics described an overwhelmingly large proportion of choices, whereas strategies traditionally deemed rational were barely apparent in our data. Replicating previous findings, we also observed that visual stimulus segments received a much larger proportion of attention than any nutritional values did. Our results suggest that, consistent with human behavior in other domains, people make their food choices on the basis of simple and informationally frugal heuristics.
\end{abstract}

(ㄷ) 2013 Elsevier Ltd. All rights reserved.

\section{Introduction}

The US stock trader Ivan Frederick Boesky is known not only for being the person who inspired the fictional character Gordon Gekko in the Oliver Stone movie Wall Street (Pressman \& Stone, 1987), but also for his eccentric food choice habits. According to Stewart (1992), at a lunch meeting in the Café des Artistes, a high-end restaurant in Manhattan, Boesky ordered every single entrée on the menu. When the food arrived, the waiter set up a second table and Boesky looked carefully at all eight dishes, took one bite of each, chose one dish, and sent the rest back.

Whether or not one perceives this to be a decadent way of choosing food, it is certainly costly, and is hardly practicable in the many food choices people make on a daily basis. Unless attend-

\footnotetext{
7) Acknowledgements: The first two authors contributed equally to this article. This study was funded by the Nestlé Research Center, at which de Bellis and Sohn conducted the experiments during an internship. Schulte-Mecklenbeck was employed by the Nestlé Research Center as a Research Scientist. We are grateful to Susannah Goss for editing the manuscript. We thank Jutta Mata for comments on an earlier version of this draft.

* Corresponding author.

E-mail address: research@schulte-mecklenbeck.com (M. Schulte-Mecklenbeck).
}

ing a buffet dinner, consumers cannot generally afford to sample all dietary options before making a final choice. Thus, pieces of information other than actual taste need to be considered. These can be retrieved from the external environment or from memory, and may include brand information (Jacoby, Szybillo, \& BusatoSchach, 1977), nutritional values (Higginson, Rayner, Draper, \& Kirk, 2002; Van Herpen \& Van Trijp, 2011), price (De Irala-Estevez et al., 2000; Drewnowski \& Specter, 2004), and attributes such as fair trade and animal welfare (Zander \& Hamm, 2010).

A key question in research on food choice is how people use this wealth of information when choosing between dishes (Scheibehenne, Miesler, \& Todd, 2007; Wansink, Just, \& Payne, 2009). Much like Boesky wanted to sample all available entrées, decision makers obeying the commandments of rational choice are assumed to sample all available information first and then to combine it into an overall evaluation. Indeed, the prevalent view on how people make food choices today predicts such weighting and integrating approaches, in implicit or explicit form (Glanz, Basil, Maibach, Goldberg, \& Snyder, 1998; Rappoport, Peters, Downey, McCann, \& Huff-Corzine, 1993). Acknowledging the constraints of time, knowledge, and computational power under which humans make choices and decisions, Simon (1955, 1990a) 
offered an alternative vision of how the human mind operates. Given these limitations, humans 'must use approximate methods to handle most tasks' (Simon, 1990b, p. 6). These methods include simple heuristics that guide search, determine when it ends, and make use of the information obtained without processing it in a complex way (Gigerenzer, Hertwig, \& Pachur, 2011; Gigerenzer, Todd, \& The ABC Research Group, 1999; Hertwig, Hoffrage, \& The ABC Research Group, 2012; Payne, Bettman, \& Johnson, 1993; Todd, Gigerenzer, \& The ABC Research Group, 2012).

Transferring these findings to the choice of a lunch dish, we can distinguish two classes of choice strategies that people may apply to their meal choices: compensatory strategies (e.g., sample all nutritional and price information and combine that knowledge into a choice), or simple heuristics that limit search to one or a few important pieces of information (e.g., decide based on a dish's price or how attractive it looks), forgo complex computations, and make no or only simple trade-offs. ${ }^{1}$ In what follows, we briefly review past work to gauge which of these two classes of strategies may be more prevalent in food choice and then turn to the hypotheses and methodology that guide our investigation.

\section{Food choice: compensatory or non-compensatory?}

Scheibehenne et al. (2007) investigated the extent to which people rely on simple heuristics or compensatory strategies in food choice. To this end, they had people repeatedly choose between pairs of lunch dishes. Using the Food Frequency Questionnaire (Steptoe, Pollard, \& Wardle, 1995), they probed individual importance weights of food-related information based on price, sensory appeal, convenience, health, weight control, natural content, mood, familiarity, and ethical concerns. Employing these factor ratings for each person and each lunch dish, they then predicted which dish a person would prefer. Specifically, they pitted the weighted additive strategy (WADD; weighting all attributes and comparing options based on the summed weights; Payne et al., 1993) against a heuristic called the lexicographic decision rule (LEX). A person applying LEX compares the options' attributes, one at a time, choosing the option with the more attractive value on the most important attribute (e.g., selecting the dish with the lowest calories).

In Scheibehenne et al.'s (2007) investigation, LEX and WADD scored nearly equally well in predicting people's choices, making $72 \%$ and $73 \%$ correct predictions, respectively. This near identical performance suggests that a simple heuristic, relying on one important attribute, describes human food choice as well as does a compensatory strategy that requires the combination of several factors. What might explain the two strategies' similar descriptive performance? First, simple and complex strategies-despite substantial differences in information search and combination of information (or lack thereof)-often result in identical observable choices. Second, Scheibehenne et al. focused on just two strategies; it may be that other compensatory or non-compensatory strategies capture food choice even better. Indeed, research has repeatedly shown that different people tend to use different heuristics (Gigerenzer \& Gaissmaier, 2011; Pachur \& Bröder, 2013; Payne et al., 1993).

In summary, based primarily on outcome data and considering a small set of strategies, Scheibehenne et al. (2007) provided an existence proof that a heuristic that limits search can explain food

\footnotetext{
${ }^{1}$ Apart from guiding search for information about the food items themselves, heuristics can also guide search in the social environment. For instance, a person may apply the imitation heuristic (Hertwig et al., 2012) and choose what either her companion orders or the majority of people at her table order (McFerran, Dahl, Fitzsimons, \& Morales, 2010; see Todd and Minard (in press) for additional social heuristics). Alternatively, a person could order the default option (Downs, Loewenstein, \& Wisdom, 2009; Johnson \& Goldstein, 2003).
}

choices as well as a compensatory strategy can. The goal of our investigation, going beyond an existence proof, is to examine the cognitive processes that underlie food choices, thus making it possible to differentiate between strategies leading to identical observable choices. In addition, we investigate a wider set of cognitive strategies that people may use to choose between foods.

\section{Process tracing: gaining a window on search and attentional processes}

Compensatory and non-compensatory strategies involve markedly different information search processes (Pachur, Hertwig, Gigerenzer, \& Brandstätter, 2013). The non-compensatory LEX, for instance, assumes a sequential, attribute-based information-sampling process that is terminated as soon as a discriminating attribute is found. In the fastest case, LEX looks up both options' values on the most important attribute, chooses the option that best satisfies this highest ranked attribute, and ignores all other information. Search is thus limited and occurs between options. The compensatory strategy WADD, in contrast, considers all values on all attributes and renders a choice based on this overall evaluation. Search is thus complete and proceeds within options.

This qualitative difference in search processes can be exploited to identify which strategy a person applies-provided that the process of information acquisition can be made observable. Processtracing methods do exactly that: they offer a window onto the cognitive processes that result in a preference or an inference (Schulte-Mecklenbeck, Kühberger, \& Ranyard, 2011a, 2011b). Process-tracing methods come in many different forms, including thinking-aloud protocols (Ericsson \& Moxley, 2011), information boards (Willemsen \& Johnson, 2011), eye tracking (Russo, 2011), and mouse tracking (Schulte-Mecklenbeck, Murphy, \& Hutzler, 2011). All of these methods aim to enable researchers to infer a person's choice strategies from pre-decisional information search and acquisition patterns.

The process-tracing tool used in this study is MouselabWeb (Willemsen \& Johnson, 2011). Additionally to choices, it records how often an attribute is inspected. Frequency of inspection is a proxy measure for the amount of attention and, by extension, the weight of importance an attribute receives. Furthermore, MouselabWeb records how search for attributes unfolds. In this study, we combine process and outcome data (ratings of attributes) to investigate the following hypotheses.

\section{Hypotheses: how do people choose in the food domain?}

1. Strategy Hypothesis: Compensatory strategies demand substantial investment in information search and computation to render a choice. In many real world contexts, people rely-for various reasons (Gigerenzer et al., 2011)-on strategies that limit search and computation. To the extent that the domain of food choice obeys the same regularity, we hypothesize that people are more likely to rely on strategies that limit search and computation than on compensatory strategies. We test this hypothesis by mapping search direction, completeness of search, and weighting of attributes onto eight different strategies.

In addition to this central hypothesis, we aim to replicate results from the literature concerning the attention paid to visual stimuli by utilizing more precise measurements:

2. Visual Dominance Hypothesis: Past research has demonstrated that the weight given to visual information in choices and judgments about food (Scheibehenne, Todd, \& Wansink, 2010; Wansink, Painter, \& North, 2005) is higher than, for instance, that given to nutritional information (Aikman, Min, \& Graham, 2006). In line with these findings, we hypothesize that consumers rely on visual information more than on any other type of available information when choosing between dishes. 


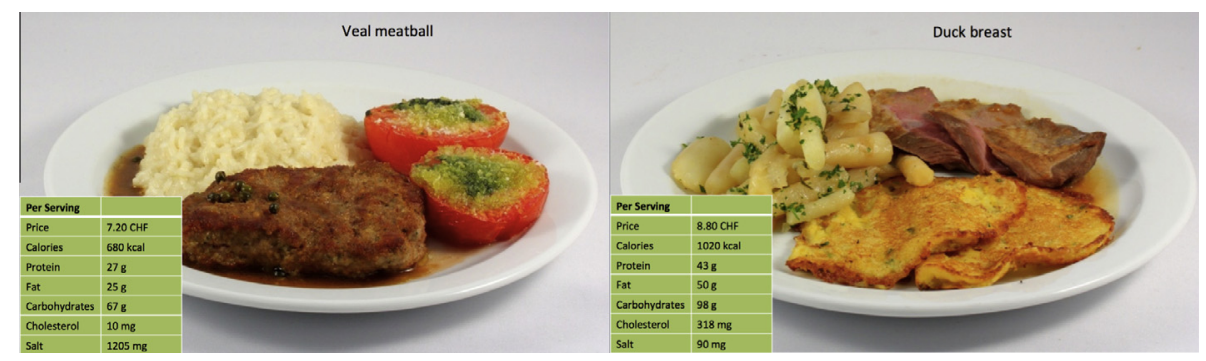

Fig. 1. Two examples of lunch dish information displayed at the entrance of the Nestlé Research Center canteen.

Table 1

Names of the 20 dishes (10 dish pairs) presented to participants (translated from French).

\begin{tabular}{cll}
\hline Pair \# & Dish 1 & Dish 2 \\
\hline 1 & Fillet of trout & Pork osso bucco \\
2 & Pork chop & Squid Portuguese style \\
3 & Fillet of mackerel & Veal Milanese \\
4 & Veal meatball & Duck breast \\
5 & Wiener schnitzel & Fillet of trout \\
6 & Rice casimir & Chicken cordon bleu \\
7 & Chicken bami goreng & Pork osso bucco with gremolata \\
8 & Duck breast & Chicken cordon bleu \\
9 & Veal Zurich style & Chicken curry \\
10 & Lamb kebab & Sausage with cabbage \\
\hline
\end{tabular}

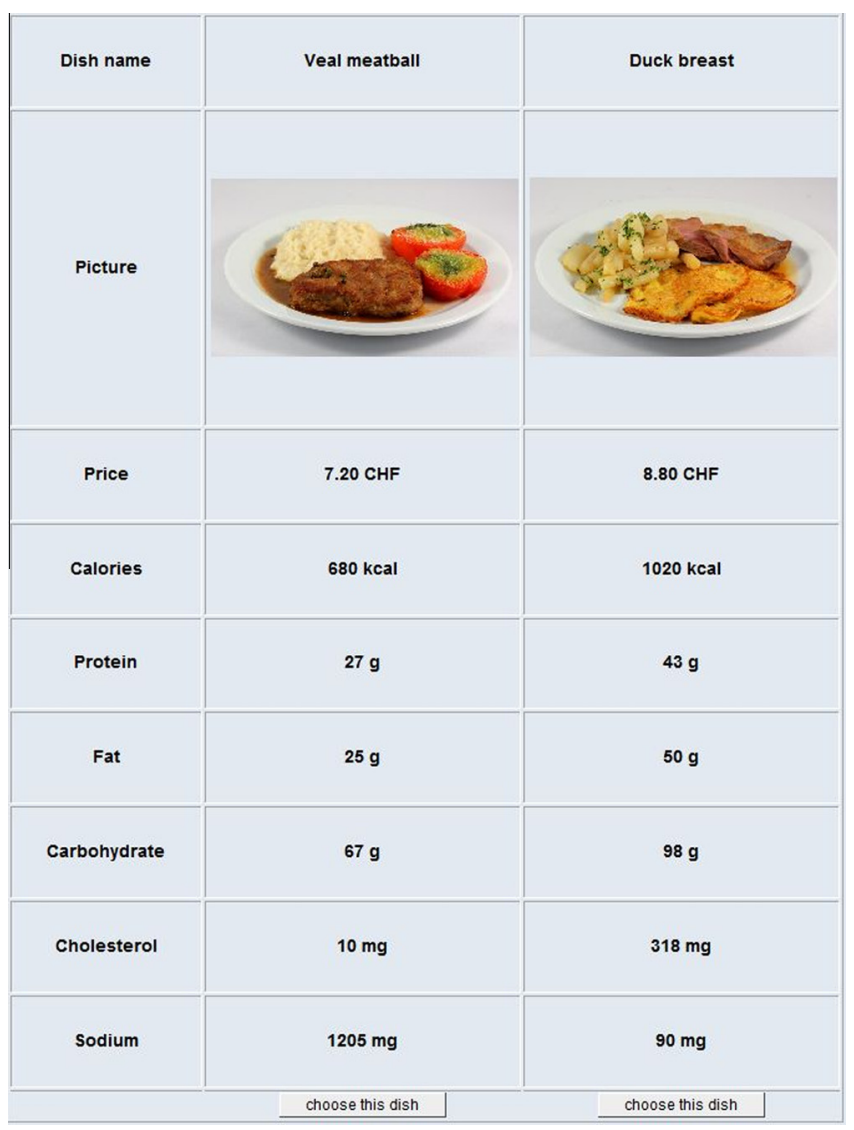

Fig. 2. Screenshot of a choice matrix provided in MouselabWeb. All cells were closed unless the cursor was moved over them. Here, all cells are opened for illustration purposes only.

\section{Method}

\section{Stimulus selection}

Dishes were sampled from a large data pool compiled in the canteen of the Nestlé Research Center. The data pool consisted of information about all 414 dishes offered in the canteen between January 2010 and June 2010, including high-resolution images, dishes' names, prices, and information on six nutritional values (calories, protein, fat, carbohydrates, cholesterol, and salt).

Figure 1 shows examples of the information displayed to guests entering the restaurant. Of the 414 dishes in the data pool, we sought to choose those 10 pairs that differed highly on the attributes considered. To this end, we calculated the Euclidian distance between nutritional values:

$d(p, q)=\sqrt{\sum_{i=1}^{n}\left(p_{i}-q_{i}\right)^{2}}$

Here, $p$ and $q$ represent the values of the attributes for the two options compared and $n$ represents the number of attributes.

In our final 20 dishes (see Table 1 for names), calories ranged from 436 to $1179 \mathrm{cal}$, fat from 9 to $63 \mathrm{~g}$, carbohydrates from 28 to $112 \mathrm{~g}$, proteins from 25 to $66 \mathrm{~g}$, cholesterol from 10 to $430 \mathrm{mg}$, and salt from 16 to $1231 \mathrm{mg}$.

\section{Stimulus presentation: MouselabWeb}

We used the open source program MouselabWeb 1.00 beta (Willemsen \& Johnson, 2011) to display the dish-specific information in a matrix form, echoing the design of food labels and the original display of information in the canteen (Fig. 1). At the beginning of each choice, all pieces of information were concealed (cf. Fig. 2, in which all cells are opened for illustration purposes only). Participants could open a cell by moving the cursor over it; the cell closed when the cursor was moved away. This presentation format is referred to as a 'closed paradigm'. The MouselabWeb matrix consisted of two columns, each representing one dish. Each column contained the dish's name, its image, its price, and six nutritional values. The position of the dishes in the columns (left/ right) was counterbalanced between choices and participants. Further, the dish's name and its image were presented in a fixed position (first two rows), while the remaining seven attributes were counterbalanced in terms of the row in which they appeared. In addition to this column setup, we created a row setup that presented the options' attributes in horizontal rows (rather than vertical columns as in Fig. 2). We thus controlled for reading direction (i.e., reading from left to right in Western cultures). ${ }^{2}$

\footnotetext{
2 The presentation format resulted in a shift of the search metric index (SM, see below) from -0.41 (vertical) to -0.15 (horizontal). However, the classification probability of strategies to the process-based metrics (search and completeness) did not differ depending on the presentation format (horizontal or vertical). Hence we combined the data from both presentation formats in our analysis.
} 
Table 2

Defining properties of eight choice strategies.

\begin{tabular}{|c|c|c|c|c|c|c|c|c|c|}
\hline Metric & \multicolumn{8}{|c|}{ Components and strategies } & \multirow{3}{*}{$\begin{array}{l}\text { Data source } \\
\text { MouselabWeb } \\
\text { MouselabWeb }\end{array}$} \\
\hline Search & \multicolumn{4}{|c|}{ Within option } & \multicolumn{4}{|c|}{ Between option } & \\
\hline Completeness & \multicolumn{2}{|l|}{ Complete } & \multicolumn{2}{|l|}{ Limited } & \multicolumn{2}{|l|}{ Complete } & \multicolumn{2}{|l|}{ Limited } & \\
\hline Weighting & Unequal & Equal & Unequal & Equal & Unequal & Equal & Unequal & Equal & Rating \\
\hline & WADD & EQW & F-WADD & F-EQW & MISC & MCD & LEX & MIN & \\
\hline
\end{tabular}

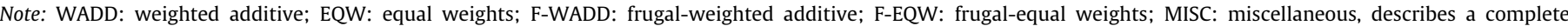

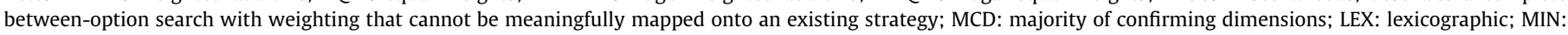
minimalist; RND: random, does not look up any information and is not listed here.

Table 3

Process description of each strategy with examples for the choice between two lunch dishes.

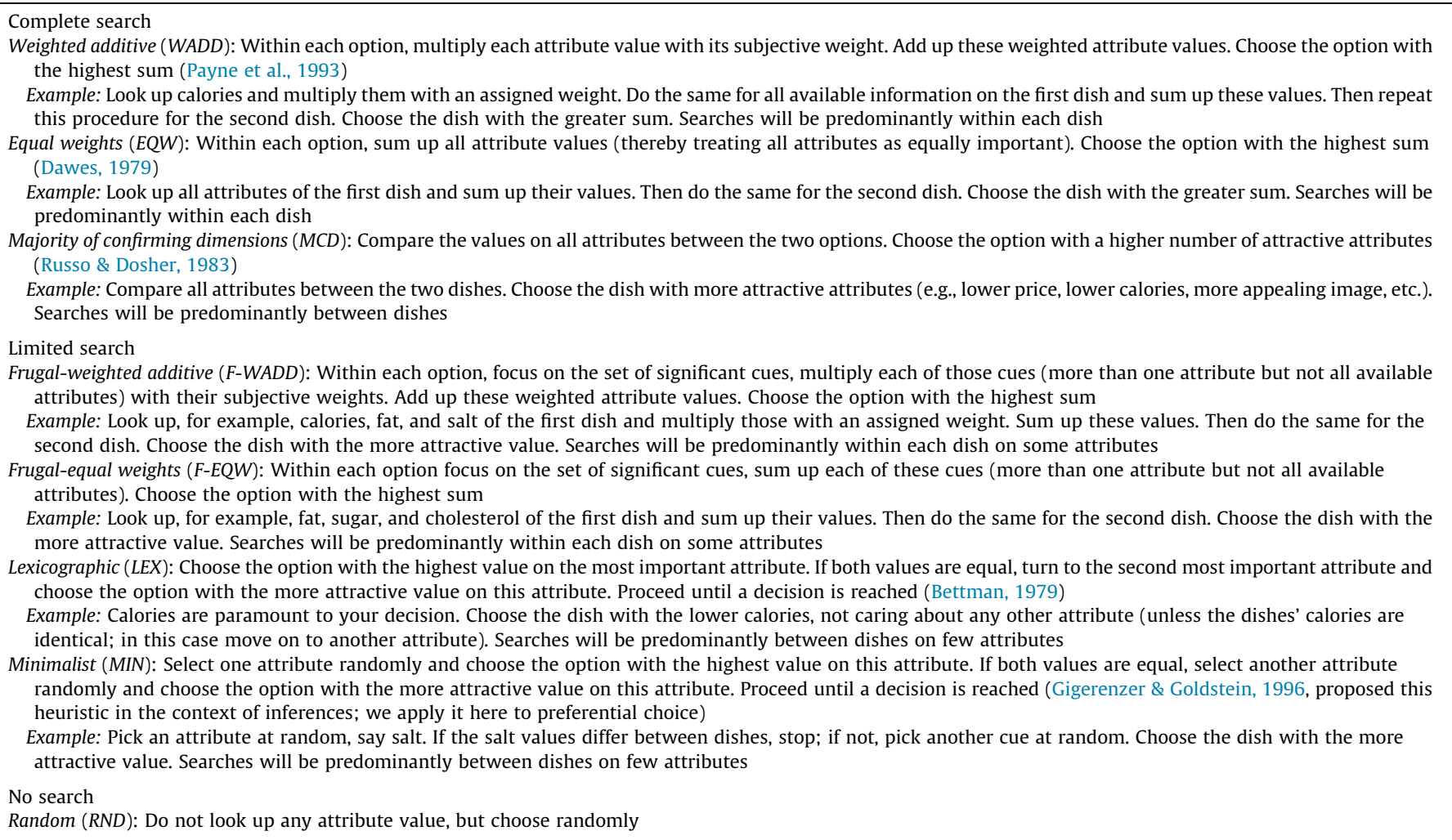

\section{Strategy classification}

Drawing on (a) the theoretically possible information acquisition patterns and (b) the theoretically possible weighting patterns of the subjective importance of attributes, we defined eight choice strategies (see Table 2). These strategies are described by three measures (called metrics; see Riedl, Brandstätter, \& Roithmayr, 2008, for a similar approach): the search metric (i.e., search unfolds mainly within or between options); the completeness metric (i.e., all or just part of the available information is looked up in the course of search), and the weighting metric (i.e., the importance of attributes is judged as being equal or different). Each metric has two levels, resulting in eight unique combinations that are associated with distinct choice strategies, as shown in Table 2.

The policies of the choice strategies are presented in detail, separately for complete and limited search, in Table 3. Each strategy is first described in an idealized version and then exemplified for our food choices for complete, limited and no search. Five of the eight strategies have previously been proposed and studied in research on behavioral decision making: WADD, EQW, MCD, LEX, and
MIN. Two of the hypothetically possible remaining strategies represent close relatives of WADD and EQW, the difference being that they forgo acquisition of all available information (being somewhat frugal in their information use; see Gigerenzer \& Todd, 1999). We refer to them as frugal-weighted additive (F-WADD) and frugalequal weights (F-EQW). In the literature on unit-weight models, variants have been discussed in which not all cues, but only the 'right' ones are picked out (Dawes, 1979). We do not define what is 'right' here, but assume that those cues that a person looks up are important, whereas ignored cues lack importance to the decision maker. Finally, we added a random choice strategy that forgoes any search for information. Next, we describe the three metrics we used as a measurement tool in more detail.

\section{Search metric}

The calculation of the search metric (SM) was based on the assumption that strategies differ in their ratio of within- to between-option transitions. Specifically, we differentiated two basic transitions in an information matrix: (a) If two cells within one 
option were opened successively, the transition was defined as within option (WO). (b) If two cells describing the same attribute were opened successively for two different options, the transition was defined as between option (BO). Using these transition types, we calculated a ratio of within- to between-option transitions (see Böckenholt \& Hynan, 1994, for a discussion and comparison of different indices).

$\mathrm{SM}=\frac{\sqrt{N}\left(\left(\frac{A * O}{N}\right)(W O-B O)-(O-A)\right)}{\sqrt{A^{2}(O-1)+O^{2}(A-1)}}$

In Eq. (2), $O$ and $A$ represent the number of options and attributes (the setup of the matrix), $N$ denotes the total number of transitions, $W O$ and $B O$ represent the number of within-option and between-option transitions, respectively. ${ }^{3}$ An SM value above 0 denotes a within-option acquisition pattern (expected for WADD, EQW, F-WADD, and F-EQW; see Table 3 for a description of the strategies examined) and an SM value below 0 denotes a between-option acquisition pattern (expected for MCD, LEX, and MIN).

\section{Completeness metric}

Cases in which consumers acquired, at least once, 18 unique pieces of information (from two options with nine attributes each) before choosing a dish were categorized as complete (as implied by WADD, EQW, and MCD). Conversely, cases in which consumers acquired fewer than 18 pieces of information before choosing a dish were categorized as limited $^{4}$ (as implied by F-WADD, F-EQW, LEX, and MIN).

\section{Weighting metric}

Participants rated the importance of each attribute (the dish's name, image, price, calories, protein, fat, carbohydrates, cholesterol, and salt) on a scale from 1 to 100 . To classify these ratings, we used the coefficient of variation $(\mathrm{CV})$, a normalized measure of dispersion:

$\mathrm{CV}=\frac{\sigma}{\mu}$

In Eq. (3), $\mu$ represents the mean of the weights of all attributes and $\sigma$ represents the standard deviation of these weights. We set the cutoff level ${ }^{5}$ for this metric to 1 : ratios with a CV $>1$ are considered high in variance-that is, participants favor some attributes over others (as implied by WADD, F-WADD, and LEX). Those with a CV $<1$ are considered low in variance-that is, participants do not favor some attributes over others (as implied by EQW, F-EQW, MCD, and MIN).

\section{Participants}

Seventy (French-speaking) participants from Lausanne, Switzerland, were recruited. Participants in a pilot session were not included in the final analysis of 56 participants. Their mean age was 42.15 years $(S D=12.94)$ and 26 were female (two participants did not report their gender). The present study was part of a larger study, in which participants completed other, unrelated tasks after

\footnotetext{
${ }^{3}$ We replaced the absolute occurrences of transitions $N$ with proportions to capture the index's sensitivity to large Ns (see also Pachur et al., 2013, for a similar approach). Our empirical distribution of SM had a mean of $-0.34(S D=0.56)$ and ranged from -1.93 to 0.86 , indicating stronger between-option comparisons.

${ }^{4}$ Note that search can be limited with respect to the number of cues to be checked (as in compensatory strategies) or the number of differences in cue values until a discriminating cue is found. Here the number of cues can be as small as one (as in, e.g., LEX). We thank an anonymous reviewer for pointing this out.

${ }^{5}$ See the sensitivity analysis in the Results section for a variation of this cutoff.
}

rendering the food choices. Participants received CHF 70 (approximately USD 75) for participating in the 2-hour-long study.

\section{Procedure}

To ensure a comparable level of appetite, we instructed participants to have their regular breakfast at least $2 \mathrm{~h}$ prior to the study (and then to fast, consuming nothing but water). All participants reported having followed this instruction. Each trial started at 11 a.m. in order to minimize differences in blood glucose levels, which typically vary according to several factors such as food intake during the day (Mayer, 1955). Participants first signed the consent form and drank a glucose drink, which contained mineral water and $50 \mathrm{~g}$ of glucose. The drink was prepared shortly before each trial, and participants were not given information about the content of the drink. Together with the instruction to fast, this initial glucose drink aimed to create similar blood glucose levels among participants and to put them into a state of not being hungry. This is relevant because differences in blood glucose may influence food choice (Hoefling \& Strack, 2010). Moreover, the importance of food attributes can change when consumers are hungry. Participants' average self-reported hunger rating, as tapped immediately before the food choice ('How hungry are you right now?') on a 9-point Likert scale ( $1=$ not hungry at all to $9=$ very hungry), was $2.69(S D=2.04)$.

Participants then read detailed instructions about how to use MouselabWeb and conducted several practice trials. The information acquisition and food choices were run for 12 pairs of dishes (10 unique pairs plus repetitions of the first two pairs to check for consistency). Finally, participants were asked to rate how (subjectively) important the nine attributes were for their food choices.

\section{Results}

\section{Test of strategy use}

We hypothesized that in food choice, like in many other real world domains (Gigerenzer et al., 2011), people are more likely to rely on strategies that limit search and computation than to use compensatory strategies (with complete search and complex computation). We tested this Strategy Hypothesis in a large data set. Specifically, our 56 participants made 560 unique choices, having acquired a total of 10,599 pieces of information. The average completion time for a single choice was $15.28 \mathrm{~s}(S D=8.04 \mathrm{~s})$; the range was from 3.09 to $38.47 \mathrm{~s}$. We were able to classify $98 \%$ of all search patterns as corresponding to one of our eight strategies; the remaining $2 \%$ were classified as MISC.

Table 4 ('Relative use of strategies across choices, $N=560$, cutoff $=1^{\prime}$ ) shows the relative use of each strategy with a weighting metric cutoff set to one across participants (56) and choices (10). The following observations are noteworthy: First, no decision strategy was consistent with WADD, the compensatory gold standard embodying complete search with weighting and summing of all available pieces of information. Second, no choice proved to be purely random (i.e., was made without any search). Third, 25\% of choice patterns were consistent with strategies that do not limit search, but forgo weighting (EQW and MCD). Fourth, 74\% of patterns were consistent with strategies that assume limited search (F-WADD, F-EQW, LEX, and MIN), but differ in the extent to which they incorporate weighting and summing.

The above analysis focused on strategies in choices pooled across all participants. The same picture emerged when the unit of analysis was people rather than each of the 560 choices. Specifically, we assigned each participant to the choice strategy she 'used' in the relative majority of her choices (Table 4, 'Strategy used 
Table 4

Categorization results for relative and majority use of strategies in percent.

\begin{tabular}{|c|c|c|c|c|c|c|c|c|}
\hline & \multicolumn{3}{|c|}{ Complete search } & \multicolumn{4}{|c|}{ Limited search } & \multirow{2}{*}{$\begin{array}{l}\text { No search } \\
\text { RND }\end{array}$} \\
\hline & WADD & EQW & MCD & F-WADD & F-EQW & LEX & MIN & \\
\hline Relative use of strategies across choices $(N=560$ choices, cutoff $=1)$ & 0 & 4 & 21 & 12 & 13 & 19 & 29 & 0 \\
\hline Relative use of strategies across choices $(N=560$ choices, cutoff $=0.5)$ & 2 & 2 & 14 & 18 & 7 & 32 & 16 & 0 \\
\hline Strategy used in majority of ten choices per participant $(N=56)$ & 0 & 4 & 23 & 13 & 9 & 20 & 32 & 0 \\
\hline
\end{tabular}

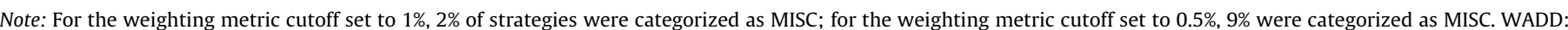

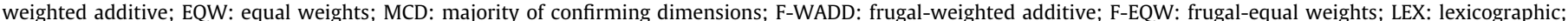
MIN: minimalist; RND: random.

in majority of ten choices per participant, $\left.N=56^{\prime}\right) .{ }^{6}$ Of the 56 participants, 41 (74\%) used strategies that limited search in the majority of their choices, $X^{2}(56)=12.07, p=.001$. Only a quarter of participants used one of the two strategies that required all information to be looked up (EQW or MCD). That is, all participants predominantly used a strategy that either looked up all information but ignored weighting (EQW and MCD) or limited search and weighted only partly, if at all (F-WADD, F-EQW, LEX, MIN). No participants predominantly summed and weighted the available information, as suggested by WADD. Even when we focused on those strategies that previous research has proposed (WADD, EQW, MCD, LEX, and MIN; thus excluding F-WADD and F-EQW), 52\% relative to $27 \%$ of participants predominantly used search patterns consistent with strategies that embody limited search.

\section{Test of allocation of attention}

Which food-related information do people pay most attention to and which tends to go unnoticed? MouselabWeb offers several indicators of people's allocation of attention: (a) how long cells are opened (duration), (b) how often cells are opened (frequency), (c) at which point in the sequence of openings cells are inspected (order), (d) which cells are opened first (first cell), and (e) which cells are opened immediately before choice (last cell). Because of the skewed distributions of frequency and duration, the data were log-transformed before further analysis. To investigate the Visual Dominance Hypothesis that visual information has priority over other kinds of information, we built a hierarchical model (using the R lmer function from the lme4 package; Bates, Mächler, \& Bolker, 2012) and included a random effect for participants and choices, thus accounting for the repeated measures in our design (Baayen, Davidson, \& Bates, 2008).

In the first analysis, we turned to duration. Specifically, we constructed a base model that predicted duration by participant and choice (each choice between two dishes) as a random effect (allowing for varying slopes). We compared this base model with a model including the factor attribute (with nine levels; see Fig. 2) as a fixed effect. We found that including attribute as a fixed factor significantly improved the model fit, $X^{2}(8)=1337, p=.001$, and reduced the BIC (Bayesian Information Criterion) from 7853 (base model) to 6582 (model including attribute). To qualify this effect, we calculated contrasts comparing the time spent on the dish's image with the time spent on the other eight attributes. We found highly significant differences between the duration for the dish image and that for the remaining eight factor levels of attributes (all ts $<-9.31$ ), indicating significantly longer durations for the dish image (see Fig. 3a).

Opening duration and frequency have commonly been interpreted as proxies for the subjective weight that information re-

\footnotetext{
${ }^{6}$ Although six participants used the exact same strategy in all choices, the majority used two $(N=36)$ or three $(N=11)$ different strategies, and none used more than four strategies. When we refer to 'use' of a strategy, here and throughout the article, this is shorthand for behavior consistent with a classification based on the combination of the three metrics described in Table 2.
}

ceives (Payne, Bettman, \& Johnson, 1988; Wedell \& Senter, 1997). We therefore ran the same analysis with frequency as dependent variable. This analysis resulted in essentially the same findings, with a significant improvement in model fit, $X^{2}(8)=835, p=.001$, when attribute was added as a fixed factor, and the dish image being opened significantly more often than any other attribute, all $t \mathrm{~s}<-15.71$ (see Fig. 3b). Most cells were opened on average 8.5 times, but the dish images were inspected, on average, close to 10 times. That is, people appeared to assign higher importance to the information encapsulated in the dish image ${ }^{7}$ than to nutritional information or price.

Another proxy for the subjective importance of each attribute is the order of information acquisition. An analysis with order of information acquisition as dependent variable produced the same pattern of results. Dish images were looked up first in 54\% of all choices-note that the dishes' names and not their images were always listed first in the MouselabWeb matrix-relative to names (27\%), prices (8\%), and all nutritional information combined (11\%). The dish image was also the cell opened immediately before choice in $28 \%$ of all choices, relative to $8 \%$ for names, $13 \%$ for price, and $51 \%$ for all nutritional information combined.

All three Mouselab indicators of attention-duration, frequency of opening, and order of acquisition-also converged with people's ratings of subjective importance. Figure $3 c$ plots importance ratings across attributes. We calculated an ANOVA with attributes as independent variables and ratings of attributes as the dependent measure and found a significant main effect, $F(1,495)=13.58$, $p=.001$, which we qualified with a contrast analysis between the dish image and the other eight attributes. These contrasts showed that the dish image was consistently rated higher in importance than the other eight attributes (all $t s<-5.01$ ). Finally, we correlated the three measures (attribute opening frequency, attribute opening duration, and attribute weights) presented in Fig. 3 and found that they were highly correlated (all $r s>.92, p s<.001$ ). This is an indication of the stability of our results across two different measurement modes (process and weighting).

In sum, despite the large amount of factual information (name, price, and nutritional information) offered to our participants, they relied most strongly on visual information in terms of both attention allocated and subjective ratings provided, consistent with previous findings (Wansink, 2007).

\section{Consistency of choices}

We next examined consistency of choices-that is, whether the same decision on pairs of dishes presented a second time would result in the same choices. To this end, we presented the first two choices again after participants had made their initial ten decisions. None of our participants mentioned noticing this repetition. We found that $62 \%$ of participants made the same two selections in these two choices (with a chance level of $25 \%$ ).

\footnotetext{
${ }^{7}$ Note however, that we had to use a larger cell size for the image than for the other information cells to ensure proper resolution and visibility. This might have skewed the order and frequency of this measure.
} 

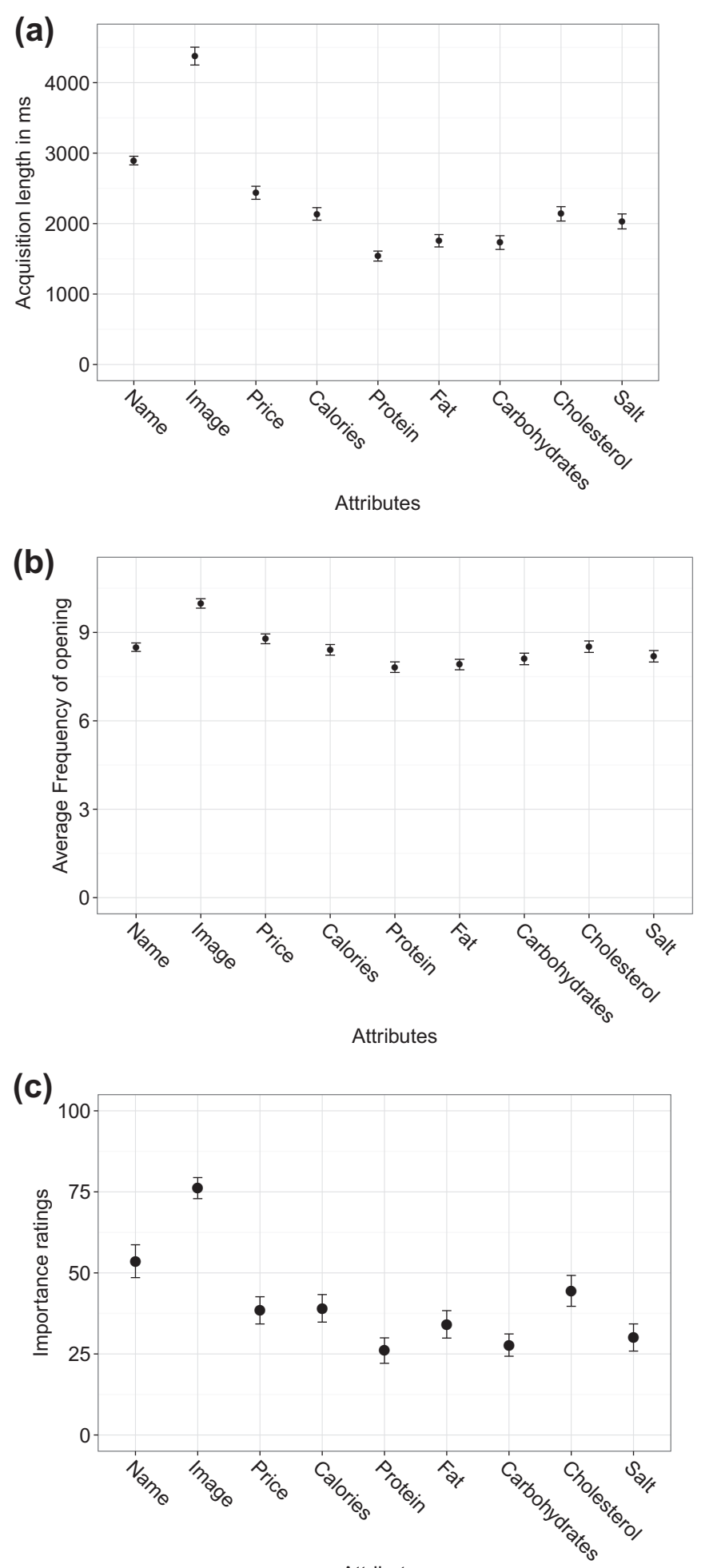

Attributes

Fig. 3. For the nine attributes: (a) Average duration of opening, (b) average frequency of opening, and (c) average importance ratings on a scale from 1 to 100 . Bars represent standard errors.

\section{Sensitivity and completion time analysis}

We inferred choice strategies from pre-decisional information search, using MouselabWeb as a process-tracing method. Models of choice strategies are idealizations; therefore, it cannot be expected that the empirically observed process patterns strictly obey these search policies. To evaluate our classification method, we first conducted a sensitivity analysis in which we relaxed the cutoff levels and observed how our classification reacted to those changes. As a second step, we repeated our classification with a new dependent variable, namely completion time, to test the basic assumption that a strategy involving complete search necessarily requires more time than a strategy that limits search.

\section{Sensitivity analysis}

Each metric in our study was dichotomized by a pre-defined cutoff value. Let us examine the weighting scheme more closely. The decision between the weighting cues equally and differently (weighting metric) was based on the coefficient of variation, set to a cutoff value of 1 . This cutoff value led to 38 cases $(67.9 \%)$ with an 'equal weighting' and 18 cases (32.1\%) with an 'unequal weighting'. Hence, it represents a high hurdle for classification as an unequal weighting. To explore the effect of relaxing the cutoff value, we reduced it by half. With the new cutoff of 0.5 , the picture was reversed: 22 cases (39.3\%) were classified as 'equal weighting' and 34 cases (60.7\%) as 'unequal weighting'. Re-running the analysis with the cutoff of 0.5 (Table 4, 'Relative use of strategies across choices, $N=560$, cutoff $=0.5^{\prime}$ ) led to classifications being changed from MIN to LEX, from F-EQW to F-WADD, and from EQW to WADD. Importantly, however, these shifts were all within either compensatory or non-compensatory strategies and did not change our main finding that non-compensatory strategies were strongly preferred over compensatory ones (73\% versus $18 \%$ ).

Still another way to evaluate the selection of our cutoff is by comparing them against the empirically observed median values, separately for each of the three metrics. The median values for search, completeness and weighting are: $-0.33,16$ and 0.6 , respectively. They are not identical with but relatively close to the ones that we chose (or have been used before, e.g., Böckenholt \& Hynan, 1994).

\section{Completion time analysis}

Our analysis of completion time was based on the idea that different choice strategies lead to different choice time predictions. Using a strategy that investigates all available information at least once (WADD, EQW, and MCD investigate all 18 information cells) will necessarily take longer than using a non-compensatory strategy that ignores part of the information (fewer than 18 cell openings; see also Scheibehenne et al., 2007, for a similar reasoning). Table 5 shows choice times based on the classification reported above. We fitted a hierarchical model to the data with strategy as a fixed effect and participants as a random effect and found a significant effect of the strategy applied on choice time, $F(6,318)=15.3, p=.001$. We qualified this effect by contrasting MCD and EQW, both assuming complete search, against strategies assuming limited search (MIN, LEX, F-EQW, and F-WADD). The latter choice times were significantly shorter than the former; specifically, the strategies relying on limited search required only half the choice times relative to MCD and EQW (all ts > 5.97). Unlike Scheibehenne et al. (2007), we thus observed substantial and theoretically expected response time differences between strategies implementing complete versus limited search. Furthermore we can qualify F-WADD and F-EQW as being equally quick as LEX and MIN and hence a member of the limited search group.

\section{Discussion}

Our goal was to investigate whether consumers indeed follow the maxim of 'more information is better' when it comes to food choice. If this were the case, our sample of participants would have engaged in an encompassing search for information, which would 
Table 5

Average choice times for each strategy in seconds.

\begin{tabular}{llll}
\hline & \multicolumn{2}{l}{ Choice times } & Search depth \\
\cline { 2 - 3 } & $M$ & $S D$ & \\
\hline MCD & 27.04 & 13.09 & Complete search \\
EQW & 24.02 & 6.38 & \\
MIN & 11.53 & 6.51 & Limited search \\
LEX & 11.42 & 7.46 & \\
F-EQW & 11.21 & 6.55 & \\
F-WADD & 10.25 & 6.15 & \\
\hline
\end{tabular}

Note: MCD: majority of confirming dimensions; EQW: equal weights; MIN: minimalist; LEX: lexicographic; F-EQW: frugal-equal weights; F-WADD: frugal-weighted additive.

then have been integrated into overall evaluations of the available options. We found the opposite. Three-quarters of all information acquisition patterns were consistent with various strategies that assume limited search. Furthermore, people classified as using non-compensatory strategies responded faster than those classified as using compensatory strategies. We also found that participants appeared to have used simplifying strategies that either limit search (MIN, LEX, F-EQW, and F-WADD) or forgo weighting (MCD and EQW). Furthermore, in line with previous findings, participants consistently allocated most attention to the dish's image and name-the two attributes that also received the highest subjective importance ratings.

\section{Comparison with previous research}

Let us now turn to commonalities and differences between Scheibehenne et al.'s (2007) investigation-the only other systematic comparison of compensatory and non-compensatory food choice strategies of which we are aware-and our own. Scheibehenne et al. relied on outcome measures with nutrition values retrieved from memory and reaction times; in contrast, we introduced a set of process measures to classify choice behavior based on nutrition values retrieved from the environment. This approach led to markedly different strategy classifications. In Scheibehenne et al., both LEX and WADD described around 70\% of choices. In our data, no choices were classified as being based on WADD; $20-30 \%$ of choices were classified as following LEX (dependent on the weighting cutoff). Furthermore, non-compensatory strategies described our participants' choices much better than compensatory ones did. We believe that the key to these differences can be found in our use of process data, which enable a finer grained analysis and overcome the problem of identical choices being predicted by different strategies. Although on the outcome level LEX described the data about as well as WADD in Scheibehenne et al.'s study, taking advantage of process data, which are better able to capture the processes underlying choice, allowed us to rule out the use of this compensatory strategy for most of our participants. Last, but not least, let us highlight that the Scheibehenne et al. investigation and our own study are unique in the sense that the choice options were sampled from real world food ecologies. Scheibehenne and colleagues presented food descriptions from different restaurants, whereas we used a sample of stimuli from a single restaurant (Nestlé canteen). Sampling experimental stimuli from real world ecologies rather than systematically constructing them fosters the chance of observing cognitive processes that generalize beyond the lab (Dhami, Hertwig, \& Hoffrage, 2004; SchulteMecklenbeck, Pachur, Pieth, \& Hertwig, 2013).

\section{Why heuristics in food choice?}

Why do people appear to rely strongly on heuristics when making food choices? There are at least two possible reasons. The first is the ubiquity of food-related decisions. People navigate the numerous demands of life under time constraints and cannot help but rely on simple strategies-not only in the domain of food choice, but also in many other professional and recreational domains (Dhami \& Ayton, 2001; Gigerenzer et al., 2011). Relatedly, because they are faced with myriads of food decisions every day, people quickly accumulate expertise in this domain. This expertise includes knowledge of what kind of attributes they value most. Being cognizant of which pieces of information matter to them makes it possible to ignore information they care less or not at all about (Neuhouser, Kristal, \& Patterson, 1999). This is not to say that ignoring nutritional information (as many of our participants did) will necessarily result in 'good' or 'adaptive' choices (which can happen when environments present redundant cues). Nevertheless, expertise-in terms of a subjective rank ordering of information-has been demonstrated to be conducive to the use of heuristics (Garcia-Retamero \& Dhami, 2009).

\section{Limitations of our investigation}

We required our participants to make a relatively small set of 12 choices in order not to overtax their motivation (i.e., they still encountered 216 boxes filled with information) and, most importantly, not to nudge them toward the use of simple strategies. As such, the fact that both we and Scheibehenne et al. (2007) found evidence for food choice heuristics cannot simply be dismissed by arguing that Scheibehenne et al. asked their participants to make 190 decisions and may thus have unwittingly fostered the use of simplifying strategies.

In this study we aimed for high ecological realism of choice stimuli. To this end, we sampled from a large database of nutrition information coming from a real canteen. We admit, however, that the stimuli represent only one ecology (the Nestle Research Center canteen) and other canteens may have rather different dishes. In addition we could, of course, not include all the possible cues that people may bring to bear to food choices such a familiarity, current mood state, yesterday's lunch, etc. We focused on cues for which we could provide reliable and veridical data.

Binary cues (e.g., a plus or minus presented to participants as cue values) are often used in strategy classification studies (e.g., Bröder \& Schiffer, 2003; Riedl et al., 2008). We, in contrast, did not dichotomize our cues (Fig. 2). A prerequisite for dichotomization is that it can be done meaningfully. Our stimuli were set up to match the information presented in the canteen as closely as possible, including the image and name of the dishes. Neither of these cues can be dichotomized. Furthermore, it is unclear which nutritional values represent a positive versus a negative value; for some participants, a high calorie count may be attractive; for others, it makes the dish unattractive. Using this approach makes it difficult to predict choices from the different strategies we classified because they depend on clear cue directions and weights for each attribute. One possibility to overcome this difficulty would be to have participants rate each attribute's weight for each choice (similarly to Scheibehenne et al.'s approach) and additionally collect the preferred direction of cue weights for each cue dimension. However, the very process of having participants provide cue weights in the wake of each choice may interfere with the processes underlying their next choice. Because our paramount interest was to record the processes as cleanly as possible, we did not probe people's cue weights in each choice. We thus relinquished the possibility to predict choices on the basis of the processes observed. However, our process results in combination with Scheibehenne et al.'s choice results strongly converge toward the same conclusion: that food choices rely predominantly on the use of simple heuristics. 
Implications for the design of consumers' information ecology

According to our findings, people are inclined to rely on simple strategies that limit search when making food choices. In addition, our participants paid more attention to the dishes' image and name at the expense of nutritional information such as caloric and salt content. We observed these informational preferences in the context of choices among lunch dishes on display in a canteen and hence cannot infer to what extent our results generalize to other food choices. However, previous investigations (using, for instance, self-reports and eye tracking) have also concluded that many consumers are reluctant to make use of food label information (Graham, Orquin, \& Visschers, 2012; Higginson, Kirk, Rayner, \& Draper, 2002; Higginson, Rayner, et al., 2002). One implication of our findings is that, if people tend to ignore much information and are inclined to invest little time in evaluating foods (both inside and outside the laboratory; Russo \& Leclerc, 1994; Sørensen, Clement, \& Gabrielsen, 2012), then one way to aid the use of information (and heuristic decision making) is to prioritize and combine information.

Food labels can (and to some extent do) provide a wide variety of desirable information, ranging from food content to production details, the food's carbon footprint, and its origins. Obviously, it makes no sense to present this information in the form of a verbose treatise. Three key tools to avoiding overtaxing the consumer and her tight time budget are prioritization, transparency, and informational shortcuts. To the extent that people rely on simple heuristics to make food choices, they will look up limited amounts of information and ignore most of the rest. Prioritization would mean rendering the information that most people value most highly, most easily accessible (Schulte-Mecklenbeck, Mata, \& Hertwig, 2013). However, this information is not necessarily the most helpful for healthy food decisions. A second key feature is information transparency; that is, the presentation of important information in an accessible manner (Mata, Lippke, Dieckmann, \& Todd, 2011). Taking advantage of informational shortcuts in such situations-by combining pieces of information that belong to categories such as nutrition, ingredients, production method, or carbon footprint into, for instance, an overall numerical score-can further improve consumers' choice quality (see Todd, Rogers, \& Payne, 2011 for such an approach). One example of such a combination is the Nutritional Scoring System (Katz et al., 2009), an index of favorable and unfavorable nutrients in a wide range of products. By referring to this single value, a consumer can then quickly decide whether the product meets his or her aspiration level-that is, is 'good enough' or 'satisficing' (Simon, 1956, 1990a).

\section{Conclusion}

This study extends the findings of Scheibehenne et al. (2007) by investigating the processes underlying food choices. It indicates that people apply a wide repertoire of strategies to their food decisions. Many of these strategies enable the decision maker to limit search and to forgo complex computations. Understanding the strategies driving food choice can help policy makers as well as industry decision makers to design communication for healthier food choice behavior.

\section{References}

Aikman, S. N., Min, K. E., \& Graham, D. (2006). Food attitudes, eating behavior and the information underlying food attitudes. Appetite, 47(1), 111-114.

Baayen, R. H., Davidson, D. J., \& Bates, D. M. (2008). Mixed-effects modeling with crossed random effects for subjects and items. Journal of Memory and Language, 59(4), 390-412.

Bates, D., Mächler, M., \& Bolker, B. (2012). lme4. Linear mixed-effects models using S4 classes. R package version 0.999999-0. <http://CRAN.R-project.org/ package=lme4>.
Bettman, J. (1979). An information processing theory of consumer choice. Reading, MA: Addison-Wesley.

Böckenholt, U., \& Hynan, L. S. (1994). Caveats on a process-tracing measure and a remedy. Journal of Behavioral Decision Making, 7(2), 103-117.

Bröder, A., \& Schiffer, S. (2003). Bayesian strategy assessment in multi-attribute decision making. Journal of Behavioral Decision Making, 16(3), 193-213.

Dawes, R. M. (1979). The robust beauty of improper linear models in decision making. American Psychologist, 34(7), 571-582.

De Irala-Estevez, J., Groth, M., Johansson, L., Oltersdorf, U., Prattala, R., \& MartinezGonzalez, M. A. (2000). A systematic review of socio-economic differences in food habits in Europe. Consumption of fruit and vegetables. European Journal of Clinical Nutrition, 54(9), 706-714.

Dhami, M. K., \& Ayton, P. (2001). Bailing and jailing the fast and frugal way. Journal of Behavioral Decision Making, 14(2), 141-168.

Dhami, M. K., Hertwig, R., \& Hoffrage, U. (2004). The role of representative design in an ecological approach to cognition. Psychological Bulletin, 130, 959-988.

Downs, J. S., Loewenstein, G., \& Wisdom, J. (2009). Strategies for promoting healthier food choices. American Economic Review, 99(2), 159-164.

Drewnowski, A., \& Specter, S. E. (2004). Poverty and obesity. The role of energy density and energy costs. The American Journal of Clinical Nutrition, 79(1), 6-16.

Ericsson, K. A., \& Moxley, J. H. (2011). Thinking aloud protocols. Concurrent verbalizations of thinking during performance on tasks involving decision making. In M. Schulte-Mecklenbeck, A. Kühberger, \& R. Ranyard (Eds.), A handbook of process tracing methods for decision research (pp. 89-114). New York, NY: Psychology Press.

Garcia-Retamero, R., \& Dhami, M. K. (2009). Take-the-best in expert-novice decision strategies for residential burglary. Psychonomic Bulletin and Review, 16(1), $163-169$.

Gigerenzer, G., \& Gaissmaier, W. (2011). Heuristic decision making. Annual Review of Psychology, 62, 451-482.

Gigerenzer, G., \& Goldstein, D. G. (1996). Reasoning the fast and frugal way. Models of bounded rationality. Psychological Review, 103(4), 650-669.

Gigerenzer, G., Hertwig, H., \& Pachur, T. (2011). Introduction. In G. Gigerenzer, R. Hertwig, \& T. Pachur (Eds.), Heuristics. The foundations of adaptive behavior (pp. 17-23). New York, NY: Oxford University Press.

Gigerenzer, G., \& Todd, P. M. (1999). Fast and frugal heuristics. The adaptive toolbox. In G. Gigerenzer, P. M. Todd, \& The ABC Research Group (Eds.), Simple heuristics that make us smart (pp. 3-34). New York, NY: Oxford University Press.

Gigerenzer, G., Todd, P. M., \& The ABC Research Group (1999). Simple heuristics that make us smart. New York, NY: Oxford University Press.

Glanz, K., Basil, M., Maibach, E., Goldberg, J., \& Snyder, D. (1998). Why Americans eat what they do. Taste, nutrition, cost, convenience and weight control concerns as influences on food consumption. Journal of the American Dietetic Association, 98(10), 1118-1126.

Graham, D. J., Orquin, J. L., \& Visschers, V. H. M. (2012). Eye tracking and nutrition label use. A review of the literature and recommendations for label enhancement. Food Policy, 37(4), 378-382.

Hertwig, R., Hoffrage, U., \& The ABC Research Group (2012). Simple heuristics in a social world. New York, NY: Oxford University Press.

Higginson, C. S., Kirk, T. R., Rayner, M. J., \& Draper, S. (2002). How do consumers use nutrition label information? Nutrition and Food Science, 32(4), 145-152.

Higginson, C. S., Rayner, M. J., Draper, S., \& Kirk, T. R. (2002). The nutrition label Which information is looked at? Nutrition and Food Science, 32(3), 92-99.

Hoefling, A., \& Strack, F. (2010). Hunger induced changes in food choice. When beggars cannot be choosers even if they are allowed to choose. Appetite, 54(3), 603-606.

Jacoby, J., Szybillo, G. J., \& Busato-Schach, J. (1977). Information acquisition behavior in brand choice situations. Journal of Consumer Research, 3(4), 209-216.

Johnson, E. J., \& Goldstein, D. (2003). Do defaults save lives? Science, 302(5649), $1338-1339$.

Katz, D. L., Njike, V. Y., Faridi, Z., Rhee, L. Q., Reeves, R. S., Jenkins, D. J., et al (2009). The stratification of foods on the basis of overall nutritional quality. The overall nutritional quality index. American Journal of Health Promotion, 24(2), 133-143.

Mata, J., Lippke, S., Dieckmann, A., \& Todd, P. M. (2011). Meat label information. Effects of separate versus conjoint presentation on product evaluation. Journal of Applied Social Psychology, 41(8), 1947-1957.

Mayer, J. (1955). Regulation of energy intake and the body weight. The glucostatic theory and the lipostatic hypothesis. Annals of the New York Academy of Sciences, $63(1), 15-43$

McFerran, B., Dahl, D. W., Fitzsimons, G. J., \& Morales, A. C. (2010). I'll have what she's having. Effects of social influence and body type on the food choices of others. Journal of Consumer Research, 36(6), 915-929.

Neuhouser, M. L., Kristal, A. R., \& Patterson, R. E. (1999). Use of food nutrition labels is associated with lower fat intake. Journal of the American Dietetic Association, 99(1), 45-53.

Pachur, T., \& Bröder, A. (in press). Judgment: A cognitive processing perspective. Wiley Interdisciplinary Reviews: Cognitive Science. http://dx.doi.org/10.1002/ wcs. 1259.

Pachur, T., Hertwig, R., Gigerenzer, G., \& Brandstätter, E. (2013). Testing process predictions of models of risky choice: A quantitative model comparison approach. Frontiers in Psychology. http://dx.doi.org/10.3389/fpsyg.2013.00646.

Payne, J. W., Bettman, J. R., \& Johnson, E. J. (1988). Adaptive strategy selection in decision making. Journal of Experimental Psychology. Learning, Memory and Cognition, 14(3), 534-552. 
Payne, J. W., Bettman, J. R., \& Johnson, E. J. (1993). The adaptive decision maker. Cambridge, UK: Cambridge University Press.

Pressman, E. R. (Producer), \& Stone, O. (Director). (1987). Wall Street [Motion picture]. United States: Twentieth Century Fox.

Rappoport, L., Peters, G. R., Downey, R., McCann, T., \& Huff-Corzine, L. (1993) Gender and age differences in food cognition. Appetite, 20(1), 33-52.

Riedl, R., Brandstätter, E., \& Roithmayr, F. (2008). Identifying decision strategies. A process- and outcome-based classification method. Behavior Research Methods, 40(3), 795-807.

Russo, J. E., \& Dosher, B. A. (1983). Strategies for multiattribute binary choice Journal of Experimental Psychology. Learning, Memory and Cognition, 9(4), 676-696.

Russo, J. E., \& Leclerc, F. (1994). An eye-fixation analysis of choice processes for consumer nondurables. Journal of Consumer Research, 21(2), 274-290.

Russo, J. E. (2011). Eye fixations as a process trace. In M. Schulte-Mecklenbeck, A. Kühberger, \& R. Ranyard (Eds.), A handbook of process tracing methods for decision research (pp. 43-64). New York, NY: Psychology Press.

Scheibehenne, B., Miesler, L., \& Todd, P. M. (2007). Fast and frugal food choices Uncovering individual decision heuristics. Appetite, 49(3), 578-589.

Scheibehenne, B., Todd, P. M., \& Wansink, B. (2010). Dining in the dark. The importance of visual cues for food consumption and satiety. Appetite, 55(3), 710-713.

Schulte-Mecklenbeck, M., Kühberger, A., \& Ranyard, R. (2011a). The role of process data in the development and testing of process models of judgment and decision making. Judgment and Decision Making, 6(8), 733-739.

Schulte-Mecklenbeck, M. Kühberger, A, \& Ranyard, R (2011b). A handbook of process tracing methods for decision research. New York, NY: Psychology Press.

Schulte-Mecklenbeck, M., Mata, J., \& Hertwig, R. (2013). Ecological intelligent labels foster healthier food choice (in preparation).

Schulte-Mecklenbeck, M., Murphy, R. O., \& Hutzler, F. (2011). Flashlight. Recording information acquisition online. Computers in Human Behavior, 27, 1771-1782.

Schulte-Mecklenbeck, M., Pachur, T., Pieth, U., \& Hertwig, R. (2013). Low in fat = low in calories? (in preparation).

Simon, H. A. (1955). A behavioral model of rational choice. The Quarterly Journal of Economics, 69(1), 99-118.

Simon, H. A. (1956). Rational choice and the structure of the environment Psychological Review, 63(2), 129-138.
Simon, H. A. (1990a). Alternative visions of rationality. In P. K. Moser (Ed.), Rationality in action. Contemporary approaches (pp. 189-204). New York, NY: Cambridge University Press.

Simon, H. A. (1990b). Invariants of human behavior. Annual Review of Psychology, 41(1), 1-20.

Sørensen, H. S., Clement, J., \& Gabrielsen, G. (2012). Food labels. An exploratory study into label information and what consumers see and understand. The International Review of Retail, Distribution and Consumer Research, 22(1), $101-114$.

Steptoe, A., Pollard, T. M., \& Wardle, J. (1995). Development of a measure of the motives underlying the selection of food. The food choice questionnaire. Appetite, 25(3), 267-284.

Stewart, J. (1992). Den of thieves. New York, NY: Simon \& Schuster.

Todd, P. M., \& Minard, S. L. (in press). Simple heuristics for deciding what to eat. In S. D. Preston, M. Kringelbach, \& B. Knutson (Eds.), The interdisciplinary science of consumption. Cambridge, MA: MIT Press.

Todd, P. M., Gigerenzer, G., \& The ABC Research Group (2012). Ecological rationality. Intelligence in the world. New York, NY: Oxford University Press.

Todd, P. M., Rogers, Y., \& Payne, S. J. (2011). Nudging the trolley in the supermarket. How to deliver the right information to shoppers. International Journal of Mobile Human Computer Interaction, 3(2), 20-34.

Van Herpen, E., \& Van Trijp, H. C. M. (2011). Front-of-pack nutrition labels. Their effect on attention and choices when consumers have varying goals and time constraints. Appetite, 57(1), 148-160.

Wansink, B. (2007). Mindless eating. Why we eat more than we think. New York, NY: Bantam.

Wansink, B., Just, D. R., \& Payne, C. R. (2009). Mindless eating and healthy heuristics for the irrational. American Economic Review, 99(2), 165-169.

Wansink, B., Painter, J. E., \& North, J. (2005). Bottomless bowls. Why visual cues of portion size may influence intake. Obesity Research, 13(1), 93-100.

Wedell, D. H., \& Senter, S. M. (1997). Looking and weighting in judgment and choice. Organizational Behavior and Human Decision Processes, 70(1), 41-64.

Willemsen, M. C., \& Johnson, E. J. (2011). Visiting the decision factory. Observing cognition with MouselabWEB and other information acquisition methods. In M. Schulte-Mecklenbeck, A. Kühberger, \& R. Ranyard (Eds.), A handbook of process tracing methods for decision research (pp. 21-42). New York, NY: Psychology Press.

Zander, K., \& Hamm, U. (2010). Consumer preferences for additional ethical attributes of organic food. Food Quality and Preference, 21(5), 495-503. 